\title{
STABILITY OF STATIONARY AND PERIODIC SOLUTIONS EQUATIONS IN BANACH SPACE
}

\author{
A.YA. DOROGOVTSEV \\ Kiev University, Mechanics and Mathematics Department \\ Vladimirskaya 64, 252033 Kiev-33 Ukraine
}

(Received April, 1996; Revised March, 1997)

\begin{abstract}
Linear difference and differential equations with operator coefficients and random stationary (periodic) input are considered. Conditions are presented for the mean stability of stationary (periodic) solutions under small perturbation of the coefficients.

Key words: Abstract Linear Equations, Stationary and Periodic Solutions, Stability, Perturbations of Coefficients.

AMS subject classifications: $60 \mathrm{H} 15,60 \mathrm{H} 20,34 \mathrm{E} 10,34 \mathrm{G} 20,34 \mathrm{~K} 30$.
\end{abstract}

\section{Introduction}

The purpose of this paper is to study the stability of stationary or periodic in law solutions for the linear difference and differential equations in Banach space under small perturbation of coefficient-operators. The problem of stability of solutions for stochastic equations is studied intensively by different methods and for various dynamical stochastic systems. See Khasminskii [9] about the pioneering results and Khaminskii and Mandrekar [10], Arnold and Khasminskii [1], Baladi and Young [2], Hinrichsen and Pritchard [8] and Wirth and Hinrichsen [14] for modern methods, new results and more references. Our results are similar to Maslow [12], which are about stability of the solution of a Cauchy problem for operator equation in Banach space. We will also need some results of [3] concerning the existence and structure of stationary and periodic solutions of operator equations in Banach space. We consider stability of solutions in the mean on $\mathbb{Z}$ or on $\mathbb{R}$ and we deal only with bounded perturbation.

\section{Assumptions}

Let $(B,\|\cdot\|)$ be complex separable Banach space, $\overline{0}$ be the zero element in $B$, and $L(B)$ be the Banach space of bounded linear operators on $B$ with the operator norm, also denoted by $\|\cdot\|$. For the function $x: \mathbb{R} \rightarrow B$, the continuity at a point $t_{0}$ means that

$$
\left\|x(t)-x\left(t_{0}\right)\right\| \rightarrow 0, \quad t \rightarrow t_{0}
$$


Function $x$ is differentiable at a point $t_{0} \in \mathbb{R}$ if there is an element $y \in B$ such that

$$
\left\|\frac{x(t)-x\left(t_{0}\right)}{t-t_{0}}-y\right\| \rightarrow 0, \quad t \rightarrow t_{0} .
$$

The element $y$ is called the derivative of function $x$ at the point $t_{0}$ and is denoted by symbol $x^{\prime}\left(t_{0}\right)$. With the help of these definitions, the classes $C(\mathbb{R}, B)$ and $C^{1}(\mathbb{R}, B)$ are defined by the usual manner. Let $\sigma(A)$ be spectrum of operator $A \in L(B)$. Denote $S:=\{z \in \mathbb{C}|| z \mid=1\}$.

In this paper we consider only $B$-valued random processes with discrete time parameter $\{x(n): n \in \mathbb{Z}\}$ or with continuous time parameter $\{x(t): t \in \mathbb{R}\}$, which is continuous on $\mathbb{R}$. For random elements and various concepts of convergence of random elements see [11]. The equality of random elements is always the equality with probability 1 . The solution of a differential equation is a $B$-valued random process $\{x(t): t \in \mathbb{R}\}$ with continuous derivative $\left\{x^{\prime}(t): t \in \mathbb{R}\right\}$. The uniqueness of the solution is within stochastic equivalence.

The $B$-valued process $\{x(n): n \in \mathbb{Z}\}$ or $\{x(t): t \in \mathbb{R}\}$ is called $\tau$-periodic with period $\tau \in \mathbb{N}$ or $\tau>0$ if all finite-dimensional distributions are periodic with period $\tau$ in time shift. For details see [3].

\section{Difference Equations}

Theorem 1: Let operators $A \in L(B)$ and $\left\{A_{m}(n), n \in \mathbb{Z}, m \geq 1\right\} \subset L(B)$ satisfy the conditions

(i) $\quad \sigma(A) \cap S=\emptyset$;

(ii) $\quad \delta_{m}:=\sup \left\{\left\|A_{m}(n)-A\right\| \mid n \in \mathbb{Z}\right\} \rightarrow 0, \quad m \rightarrow \infty$.

Then,

$$
x(n+1)=A x(n)+y(n), \quad n \in \mathbb{Z},
$$

and for every $m$ greater than some $m_{0} \in \mathbb{N}$,

$$
x_{m}(n+1)=A_{m}(n) x_{m}(n)+y(n), \quad n \in \mathbb{Z},
$$

has a unique stationary solution $\{x(n): n \in \mathbb{Z}\}$ and unique solution $\left\{x_{m}(n): n \in \mathbb{Z}\right\}$, respectively, for which

$$
E\|x(0)\|<+\infty \sup _{n \in \mathbb{Z}} E\left\|x_{m}(n)\right\|<+\infty, n \in \mathbb{Z}
$$

and

$$
\sup _{n \in \mathbb{Z}} E\left\|x_{m}(n)-x(n)\right\| \rightarrow 0, \quad m \rightarrow \infty
$$

for each stationary $B$-valued process $\{y(n): n \in \mathbb{Z}\}$ with $E\|y(0)\|<+\infty$.

Remark: Theorem 1 in 3.1 .1 in [3] states that condition $(i)$ of Theorem 1 is equivalent to the existence of a unique stationary solution $\{x(n): n \in \mathbb{Z}\}$ with $E\|x(0)\|<+\infty$ of equation (1) for every stationary process $\{y(n): n \in \mathbb{Z}\}$ with $E\|y(0)\|<+\infty$.

Proof of Theorem 1: Let $\sigma_{-}(A):=\sigma(A) \cap\{z \in \mathbb{C}|| z \mid<1\}, \sigma_{+}(A):=$ 
$\sigma(A) \backslash \sigma_{-}(A)$ and let $P_{-}$and $P_{+}$be spectral projectors corresponding to spectral sets $\sigma_{-}(A)$ and $\sigma_{+}(A)$, respectively. As proved in [3], for every $n \in \mathbb{Z}$, we have $x(n)=\sum_{j=-\infty}^{+\infty} G_{j} y(n-j-1)$, where

$$
G_{j}:=\left(A P_{-}\right)^{j} I_{(j \geq 0)}(j)-\left(A P_{+}\right)^{j} I_{(j \leq-1)}(j), j \in \mathbb{Z} .
$$

The above series expansion of $x(n)$ is convergent with probability 1 in norm $B$ and $E\|x(0)\|<+\infty$. Moreover, $L:=\sum_{j \in \mathbb{Z}}\left\|G_{j}\right\|<+\infty$.

Let $m_{0} \in \mathbb{Z}$ such that $L \delta_{m}<1$ for $\stackrel{j \in m}{m}>m_{0}$ and let $m>m_{0}$. We prove the existence of a solution for equation (2) by showing that the sequence

$$
\begin{gathered}
x_{m}^{0}(n):=\overline{0}, \quad n \in \mathbb{Z}, \\
x_{m}^{j+1}(n+1):=A x_{m}^{j+1}(n)+\left(A_{m}(n)-A\right) x_{m}^{j}(n)+y(n), n \in \mathbb{Z} ; j \geq 0,
\end{gathered}
$$

converges as $j \rightarrow \infty$ to a solution of (2). First we have

$$
\Delta_{m}^{j} \leq L \delta_{m} \Delta_{m}^{j-1}, \quad j \geq 1
$$

for $\Delta_{m}^{j}:=\sup _{n \in \mathbb{Z}} E\left\|x_{m}^{j+1}(n)-x_{m}^{j}(n)\right\|$. From [11], for every $n \in \mathbb{Z}$, there is a random element $x_{m}(n)$ such that $x_{m}^{j}(n) \rightarrow x_{m}(n), \quad j \rightarrow \infty$ with probability 1 . In addition, sup $\sup E\left\|x_{m}(n)\right\|<+\infty$ and taking the limit in $j$ in both sides of equality (4) we $n \in \mathbb{Z}$

obtain equation (3). From (1) and (2) it follows that

$$
\sup _{n \in \mathbb{Z}} E\left\|x(n)-x_{m}(n)\right\| \leq L \delta_{m} E\left\|x_{m}(n)\right\|
$$

and

$$
\sup _{n \in \mathbb{Z}} E\left\|x(n)-x_{m}(n)\right\| \leq \frac{L \delta_{m}}{1-L \delta_{m}} E\|y(0)\| .
$$

Theorem 1 is proved.

Remarks: 1 . Theorem 1 may be generalized to encompass more general perturbations. Let

and

$$
\left\{A_{m}^{\nu}(n) \mid n \in \mathbb{Z}, \nu \geq 0, m \geq 1\right\} \subset L(B)
$$

$$
\delta_{m}^{\prime}:=\sup _{n \in \mathbb{Z}}\left\|A_{m}^{0}(n)-A\right\|+\sum_{\nu=1}^{\infty} \sup _{n \in \mathbb{Z}}\left\|A_{m}^{\nu}(n)\right\| \rightarrow 0, \quad m \rightarrow \infty .
$$

Then the conclusion of Theorem 1 is valid for the solutions of the equations

$$
x_{m}(n+1)+\sum_{\nu=0}^{\infty} A_{m}^{\nu}(n) x_{m}(n-\nu)+y(n), n \in \mathbb{Z} ; m \geq 1 .
$$

2. All processes, which occurred in Theorem 1, are stationary connected processes.

Let $\{A(n): n \in \mathbb{Z}\} \subset L(B)$ and, for a fixed $\tau \in \mathbb{N}$, let $A(n+\tau)=A(n), n \in \mathbb{Z}$. 
Define $B:=A(\tau-1) A(\tau-2) \ldots A(1) A(0)$.

Theorem 2: Let operators $\{A(n): n \in \mathbb{Z}\}$ and $\left\{A_{m}(n), n \in \mathbb{Z}<m \geq 1\right\} \subset L(B)$ satisfy the conditions

(i) $\quad \sigma(B) \cap S=\emptyset$;

(ii) $\sup _{n \in \mathbb{Z}}\left\|A_{m}(n)-A(n)\right\| \rightarrow 0, \quad m \rightarrow \infty$.

Then,

$$
x(n+1)=A(n) x(n)+y(n), \quad n \in \mathbb{Z}
$$

and for every $m$ greater some $m_{0} \in \mathbb{N}$ the equation

$$
x_{m}(n+1)=A_{m}(n) x_{m}(n)+(n), \quad n \in \mathbb{Z}
$$

has a unique $\tau$-periodic solution $\{x(n): n \in \mathbb{Z}\}$ and unique solution $\left\{x_{m}(n): n \in \mathbb{Z}\right\}$, respectively, for which

$$
E\|x(k)\|<+\infty, \quad k=1,2, \ldots, \tau ; \sup _{n \in \mathbb{Z}} E\left\|x_{m}(n)\right\|<+\infty
$$

and

$$
\sup _{n \in \mathbb{Z}} E\left\|x_{m}(n)-x(n)\right\| \rightarrow 0, m \rightarrow \infty ; n \in \mathbb{Z}
$$

for each $\tau$-periodic process $\{y(n): n \in \mathbb{Z}\}$ with $E\|y(k)\|<+\infty, k=1,2, \ldots, \tau$.

Proof: The proof of Theorem 2 is similar to that of Theorem 1 and we give only new arguments. First, notice that for each process $\{y(n): n \in \mathbb{Z}\}$ with sup $E\|y(n)\|<+\infty$, equation (1) under condition $(i)$ of Theorem 1 and condition $n \in \mathbb{Z}$

(ii) of Theorem 2 has a unique solution $\{x(n): n \in \mathbb{Z}\}$ with $\sup E\|x(n)\|<+\infty$. $n \in \mathbb{Z}$

The proof of this statement follows along the lines of proof of Theorem 1 .

It is easily seen that the solution $\{x(n): n \in \mathbb{Z}\}$ for equation (6) satisfies the equation

with

$$
x((\nu+1) \tau)=B x(\nu \tau)+z(\nu), \quad \nu \in \mathbb{Z}
$$

$$
z(\nu):=\sum_{t=1}^{\tau-1} A(\tau-1) A(\tau-2) \ldots A(\tau-t) y((\nu+1) \tau-t-1)+y((\nu+1) \tau-1), \nu \in \mathbb{Z} .
$$

Then, using the previous statement, we define $\{x(\nu \tau): \nu \in \mathbb{Z}\}$ as a solution for equation (8) and with

$$
\begin{gathered}
x(\nu \tau+1):=A(0) x(\nu \tau)+y(\nu \tau), \\
x(\nu \tau+2):=A(1) x(\nu \tau+1)+y(\nu \tau+1), \\
\ldots \ldots \ldots \ldots \ldots \cdots \cdots \\
x(\nu \tau+\tau-1):=A(\tau-2) x(\nu \tau+\tau-2)+y(\nu \tau+\tau-2),
\end{gathered}
$$

we have the solutions $\{x(n): n \in \mathbb{Z}\}$ of equation (6).

Now, using the approximating method of Theorem 1 , it is easy to prove the existence of solution to equation (7) for every $m$ greater than some $m_{0} \in \mathbb{N}$. Theorem 2 is proved. 


\section{Differential Equations with Random Forces}

Theorem 3: If operators $\left\{A ; A_{m}(t) \mid m \geq 1, t \in \mathbb{R}\right\} \subset L(B)$ satisfy the following conditions

(i) $\quad \sigma(A) \cap i \mathbb{R}=\emptyset$;

(ii) $\forall m \geq 1: A_{m} \in C(\mathbb{R}, L(B))$; then

(iii) $\quad \int_{\mathbb{R}}\left\|A_{m}(t)-A\right\|^{2} d t \rightarrow 0, m \rightarrow \infty$,

$$
x^{\prime}(t)=A x(t)+\xi(t), \quad t \in \mathbb{R}
$$

and for every $m$ greater than some $m_{0} \in \mathbb{N}$, the equation

$$
x_{m}^{\prime}(t)=A_{m}(t) x_{m}(t)+\xi(t), \quad t \in \mathbb{R}
$$

has a unique stationary solution $\{x(t): t \in \mathbb{R}\}$ and unique solution $\left\{x_{m}(t): t \in \mathbb{R}\right\}$, respectively, with

$$
E\|x(0)\|<+\infty ; \sup _{t \in \mathbb{R}} E\left\|x_{m}(t)\right\|<+\infty
$$

and

$$
\sup _{t \in \mathbb{R}} E\left\|x(t)-x_{m}(t)\right\| \rightarrow 0, m \rightarrow \infty
$$

for each stationary process $\{\xi(t): t \in \mathbb{R}\}$ with $E\|\xi(0)\|<+\infty$.

Proof: In [3], 7.1.1 it was shown that condition $(i)$ of Theorem 3 is equivalent to the existence of a unique stationary solution $\{x(t): t \in \mathbb{R}\}$ to equation (9) with $E\|x(0)\|<+\infty$ for each stationary process $\{\xi(t): t \in \mathbb{R}\}$ with $E\|\xi(0)\|<+\infty$. Moreover, with probability 1 for every $t \in \mathbb{R}$,

$$
x(t)=\int_{\mathbb{R}} G(t-s) \xi(s) d s,
$$

where $G(t):=-e^{A t} P_{+} I_{(t<0)}(t)+e^{A t} P_{-} I_{(t>0)}(t), \quad t \in \mathbb{R}$ with spectral projectors $P_{-}$and $P_{+}$corresponding to spectral sets $\sigma(A) \cap\{z \mid \operatorname{Rez}<0\}$ and $\sigma(A) \cap$ $\{z \overline{\mid} \operatorname{Re} z>0\}$, respectively. The integral in (11) is a Bochner integral [15] with respect to Lebesgue measure on $\mathbb{R}$. It is known that $\|G(t)\| \leq L e^{-a|t|}, \quad t \in \mathbb{R}$ with some $L \geq 0, a>0$. In similar way, we can prove the existence of a unique solution $\left\{x_{m}(t): t \in \mathbb{R}\right\}$ of equation (10) for $m$ sufficiently large. Moreover, for each $t \in \mathbb{R}$,

and

$$
x_{m}(t)=\int_{\mathbb{R}} G(t-s)\left(A_{m}(t)-A\right) x_{m}(s) d s+x(t), \quad m \geq m_{0}
$$

$$
\sup _{m \geq 1} \sup _{t \in \mathbb{R}} E\left\|x_{m}(t)\right\|<+\infty .
$$

Then the conclusion of Theorem 3 follows from (11), (12), and condition (iii).

The following theorem is a consequence of Theorem 2. The proof is similar so that of Theorem 3 and is omitted. Let

$$
A \in C(\mathbb{R}, L(B)) ; A(t+\tau)=A(t), t \in \mathbb{R}
$$

and let $U: \mathbb{R} \rightarrow L(B)$ be an invertible valued solution to the problem 


$$
\left\{\begin{array}{c}
U^{\prime}(t)-A(t) U(t), t \in \mathbb{R} \\
U(0)=I
\end{array}\right.
$$

where $I$ is the identity operator, see [13].

Theorem 4: Let operators $\left\{A, A_{m}\right\} \subset C(\mathbb{R}, L(B)), \quad m \geq 1$ satisfy the following conditions

(i) $\quad A(t+\tau)=A(t), t \in \mathbb{R}$;

(ii) $\quad \sigma(U(\tau)) \cap S=\emptyset$;

(iii) $\quad \int_{\mathbb{R}}\left\|A(t)-A_{m}(t)\right\|^{2} d t \rightarrow 0, m \rightarrow \infty$.

Then, $x^{\prime}(t)=A(t) x(t)+\xi(t), t \in \mathbb{R}$, and for every sufficiently large $m$ the equation

$$
x_{m}^{\prime}(t)=\left(A(t)+A_{m}(t)\right) x_{m}(t)+\xi(t), \quad t \in \mathbb{R}
$$

has a unique $\tau$-periodic solution $\{x(t): t \in \mathbb{R}\}$ and $\left\{x_{m}(t): t \in \mathbb{R}\right\}$, respectively, with

and

$$
\sup _{0 \leq t \leq \tau} E\|x(t)\|<+\infty, \sup _{t \in \mathbb{R}} E\left\|x_{m}(t)\right\|<+\infty
$$

$$
\sup _{t \in \mathbb{R}} E\left\|x(t)-X_{m}(t)\right\| \rightarrow 0, \quad m \rightarrow \infty
$$

for each $\tau$-periodic process $\{\xi(t): t \in \mathbb{R}\}$ with

$$
\int_{0}^{\tau} E\|\xi(s)\| d s<+\infty .
$$

Consider also the following generalization of the last theorem.

Condition A: Let the function $A \in C(\mathbb{R}, L(B)$ have exponential dichotomy on $\mathbb{R}$ with exponent index $a>0$ and coefficient $L$ as in [7].

Theorem 5: Let operators $\left\{A, A_{m}\right\} \subset C(\mathbb{R}, L(B)), \quad m \geq 1$ satisfy the following conditions

(i) Condition $A$ for the function $A$;

(ii) $\quad \int_{\mathbb{R}}\left\|A(t)-A_{m}(t)\right\|^{2} d t \rightarrow 0, m \rightarrow \infty$.

Then, $x^{\prime}(t)=A(t) x(t)+\xi(t), t \in \mathbb{R}$ and for every sufficiently large $m$,

$$
x_{m}^{\prime}(t)=\left(A(t)+A_{m}(t)\right) x_{m}(t)+\xi(t), \quad t \in \mathbb{R}
$$

has unique solutions $\{x(t): x \in \mathbb{R}\}$ and $\left.x_{m}(t): t \in \mathbb{R}\right\}$ with

and

$$
\sup _{t \in \mathbb{R}} E\|x(t)\|<+\infty, \sup _{t \in \mathbb{R}} E\left\|x_{m}(t)\right\|<+\infty
$$

$$
\sup _{t \in \mathbb{R}} E\left\|x(t)-x_{m}(t)\right\| \rightarrow 0, \quad m \rightarrow \infty
$$

for each process $\{\xi(t): t \in \mathbb{R}\}$ with $\sup E\|\xi(t)\|<+\infty$.

$$
t \in \mathbb{R}
$$

Remarks: 1. Theorems 1-5 may be generalized to nonlinear equations, which are nearly linear as in [3]. The nonlinear equation of Riccati type [4] shall be considered in the next paper.

2. See $[5,6]$ for analogous results under some other conditions. 


\section{References}

[1] Arnold, L. and Khasminskii, R.Z., Stability index for nonlinear stochastic differential equations, Proc. of Symposia in Pure Math. 57 (1995), 543-551.

[2] Baladi, V. and Young, L.-S., On the spectra of randomly perturbed expanding maps, Comm. Math. Rhys. 156 (1993), 355-385.

[3] Dorogovtsev, A.Ya., Periodic and Stationary Regimes of Infinitely Dimensional Deterministic and Stochastic Dynamic Systems, Vischha Shkola, Kiev 1992 (in Russian).

[4] Dorogovtsev, A.Ya. and Petrova, T.A., Bound and periodic solutions of the Riccati equation in Banach space, J. Appl. Math. Stoch. Anal. 8:2 (1995), 195200.

[5] Dorogovtsev, A.Ya., Stability of periodic solutions of operator equations with perturbation coefficients, Exploring Stochastic Laws, VSP (1995), 111-119.

[6] Dorogovtsev A.Ya., Stability of bounded and stationary solutions for linear equations under perturbations operator-valued coefficients, Doklady Akad. Nauk. 2345 (1995), 488-450 (in Russian).

[7] Henry, D., Geometrical Theory of Semilinear Parabolic Equations, SpringerVerlag, Berlin 1981.

[8] Hinrichsen, D. and Pritchard, A.J., Robust Stability of Linear Evolution Operators on Banach Spaces, Report 269, Institut für Dynamische Systeme, Bremen, Germany 1992.

[9] Khasminskii, R.Z., Stochastic Stability of Differential Equations, Nauka, Moscow 1969. English Trans., Sithoff and Noordhoff, Alphen 1980.

[10] Khasminskii, R.Z. and Mandrekar, V., On Stability of Solutions of Stochastic Evolution Equations, The Dynkin Festschrift (ed. by M. Friedlin), Brikhäuser, Boston 1994.

[11] Kruglov, V.M., Supplementary Chapters of Probability Theory, Vyshtcha Shkola, Kiev 1984 (in Russian).

[12] Maslow, V.P., Asymptotical Methods and Perturbation Theory, Nauka, Moscow 1988.

[13] Massera, J.L. and Schaffer, J.J., Linear Differential Equations and Function Spaces, Academic Press, New York 1966.

[14] Wirth, F. and Hinrichsen, D., On Stability Radii of Infinite Dimensional Timevarying Discrete-time Systems, Report 276, Institut für Dynamische System, Bremen, Germany.

[15] Yosida, K., Functional Analysis, Springer-Verlag, Berlin 1965. 


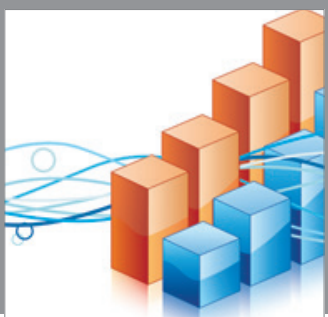

Advances in

Operations Research

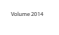

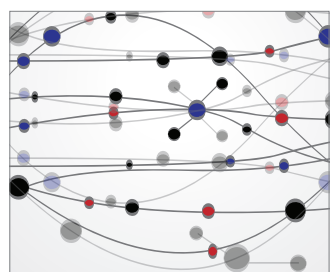

\section{The Scientific} World Journal
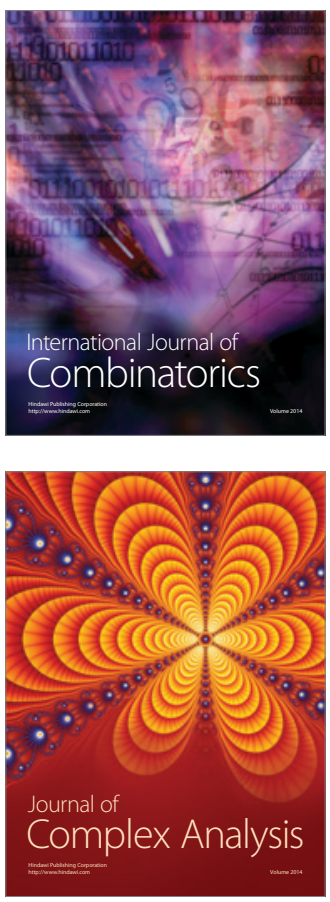

International Journal of

Mathematics and

Mathematical

Sciences
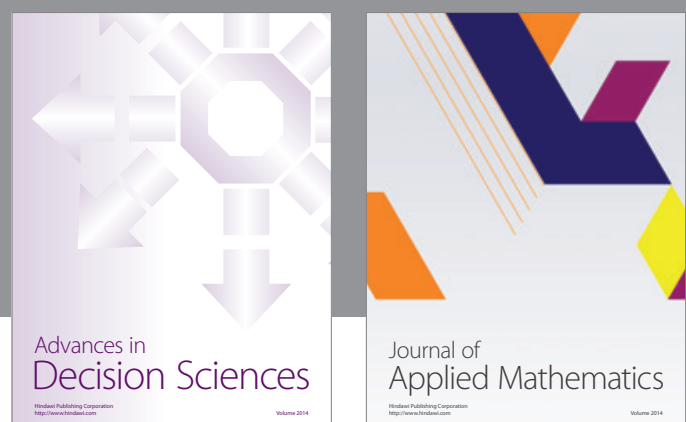

Journal of

Applied Mathematics
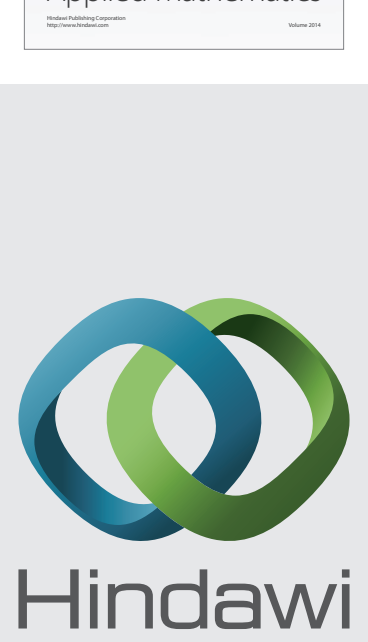

Submit your manuscripts at http://www.hindawi.com
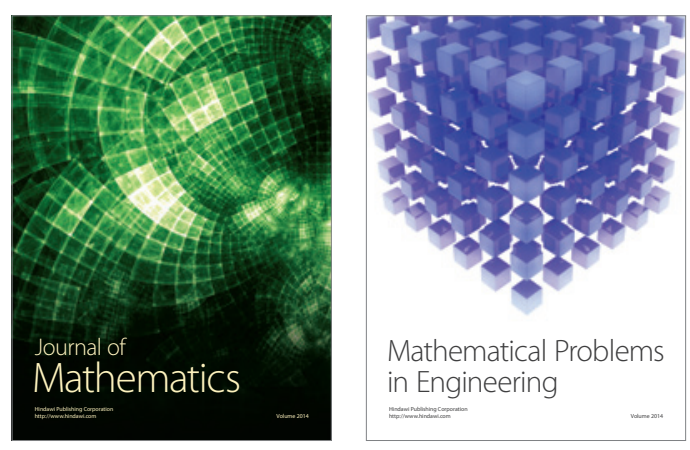

Mathematical Problems in Engineering
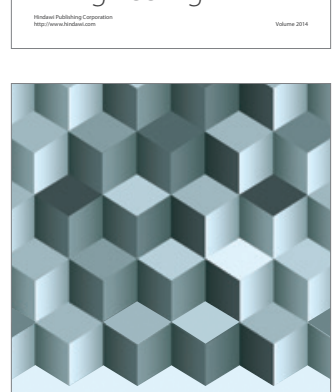

Journal of

Function Spaces
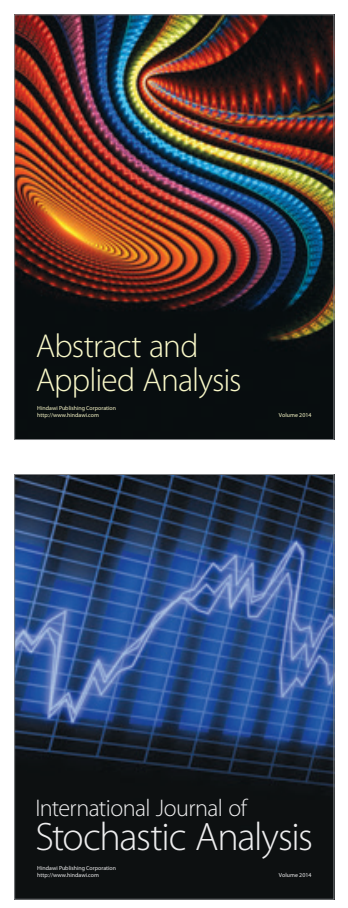

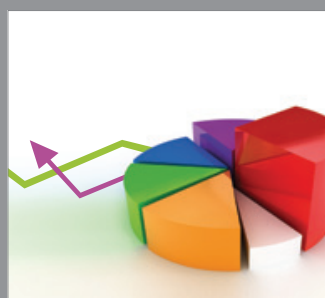

ournal of

Probability and Statistics

Promensencen
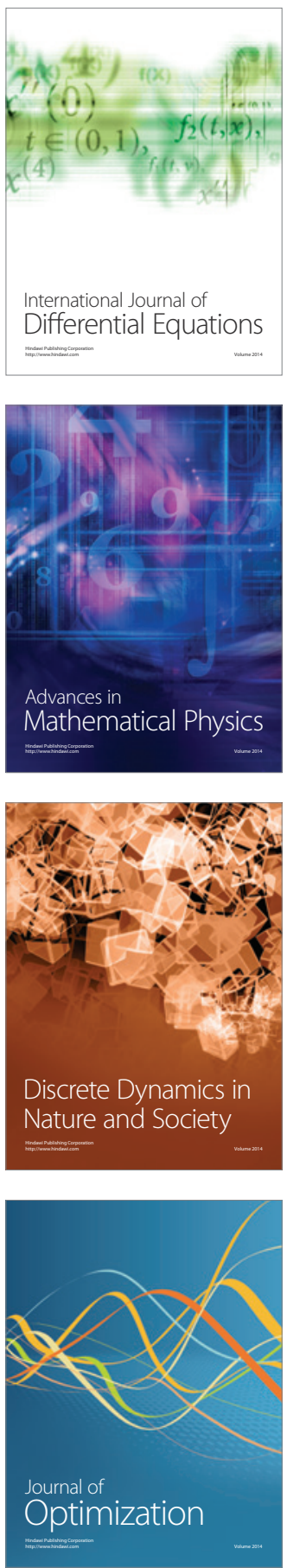